(Supporting Information)

\title{
Nonracemic Dopant-Mediated Hierarchical Amplification of Macromolecular Helicity in a Charged Polyacetylene Leading to a Cholesteric Liquid Crystal in Water
}

Katsuhiro Maeda ${ }^{\dagger}{ }^{\text {Yoshihisa Takeyama }}{ }^{\dagger}$ Koichi Sakajiri, $^{\ddagger}$ and Eiji Yashima ${ }^{* \dagger \neq}$ ${ }^{\dagger}$ Department of Molecular Design and Engineering, Graduate School of Engineering, Nagoya University, Chikusa-ku, Nagoya 464-8603, Japan, and ${ }^{\star}$ Yashima Super-structured Helix Project, ERATO, Japan Science and Technology Agency (JST), 101 Creation Core Nagoya, 2266-22 Moriyama-ku, Nagoya 463-0003, Japan

\section{Experimental Section}

Materials. Deionized distilled water was used in all the experiments. $(S)$ - and $(R)$-2 were prepared by the reaction of $(S)$ - and $(R)$-phenyl lactic acids (Aldrich) with aqueous $\mathrm{NaOH}$. The stereoregular cis-transoidal poly-1 was prepared by the polymerization of the corresponding monomer with a rhodium catalyst according to the previously reported method. ${ }^{1,2}$ The resulting polymer was quantitatively converted to its $\mathrm{HCl}$ salt with aqueous $\mathrm{HCl}$, followed by precipitation into acetone; its number average molecular weight was $3.4 \times 10^{5}\left(M_{\mathrm{w}} / M_{\mathrm{n}}=2.21\right)$ as determined by size exclusion chromatography (SEC). The stereoregularity was confirmed to be cis-transoidal based on the ${ }^{1} \mathrm{H}$ NMR and Raman spectroscopies. ${ }^{1,2}$

Instruments. ${ }^{1} \mathrm{H}$ NMR spectra were taken using a Varian VXR-500S spectrometer operating at $500 \mathrm{MHz}$ for ${ }^{1} \mathrm{H}$. The Raman spectrum was taken on a Jasco RMP-CT200. An SEC measurement was performed with a Jasco PU-980 liquid chromatograph (Jasco) equipped with a RI (256 nm; Jasco RI-930) detector. A Tosoh TSK-GEL SUPER AWM-H column was connected and a water solution containing $9 \mathrm{mM}$ tartaric acid and the $0.1 \mathrm{mM}$ tartaric acid sodium salt was used as the eluent at a flow rate of $0.6 \mathrm{~mL} / \mathrm{min}$. 
The molecular weight calibration curve was obtained with poly(ethylene oxide) and poly(ethylene glycol) standards.

Absorption and CD Measurements in Dilute Solution. All absorption (Jasco V-570, Hachioji, Japan) and CD (Jasco J-725) spectra were measured in water in a 1.0-mm quartz cell at ambient temperature $\left(20-25^{\circ} \mathrm{C}\right)$. The concentration of poly-1-HCl was calculated on the basis of the monomer units and was $1.0 \mathrm{mg} / \mathrm{mL}$ in dilute solution. The molar ratio of $(S)$ - or $(R)-2$ to the monomer units of poly-1-HCl was 0.5 (Figure. S-1) and 0.1 (Figure $2 \mathrm{~B}$, inset). The $\mathrm{CD}$ titration experiments were carried out as follows. A stock solution of poly-1- $\mathrm{HCl}(2.0 \mathrm{mg} / \mathrm{mL})$ in water was prepared in a $5-\mathrm{mL}$ flask equipped with a stopcock. Stock solutions of $(S)-2(7.94,1.99,0.40$ and $0.20 \mathrm{mM})$ in water were also prepared in 5-, 25- and two 100-mL flasks, respectively. The $0.50 \mathrm{~mL}$ aliquots of the poly-1- $\mathrm{HCl}$ solution were transferred to thirteen 1-mL flasks equipped with stopcocks. Increasing amounts of the stock solution of the $(S)-2$ were added to the flasks; the molar ratios of $(S)-\mathbf{2}$ to poly-1-HCl were $0.001,0.002,0.005(0.20 \mathrm{mM}(S)-\mathbf{2}$ was used), 0.01, 0.02, $0.03(0.40 \mathrm{mM}(S)-2), 0.05$ and $0.1(1.99 \mathrm{mM}(S)-2)$ and 0.2, 0.3, 0.5, 5.0, $10(7.94 \mathrm{mM}(S)-2)$, and the resulting solutions were diluted with water to maintain the poly-1-HCl concentrations at $1.0 \mathrm{mg} / \mathrm{mL}(4.0 \mathrm{mM})$. The absorption and CD spectra were then taken for each flask to determine the changes in the CD spectra (Figure 2A, inset). The association constant $(K)$ was obtained according to the Hill equation, $\log (\mathrm{Y} /(1-\mathrm{Y}))=n \log [\mathrm{G}]+n \log K$, where $\mathrm{Y}, n,[\mathrm{G}]$ and $K$ represent the fractional saturation, the Hill coefficient, the concentration of the guest and the association constant, respectively. ${ }^{3}$ The Hill plot gave a satisfactory linear fit (correlation coefficient $>0.99$ ) with $K=1.9 \times 10^{4}\left(\mathrm{M}^{-1}\right)$ and $n=1.20$. The changes in 
the ICD intensity of poly-1-HCl with respect to the ee of $2(5 \%<$ ee $<100 \%$, nonlinear effects) were investigated as follows. The molar ratio of 2 to the monomer units of poly-1-HCl was held constant at 0.1 . Stock solutions of the $(S)-$ and $(R)-2(0.93$ $\mathrm{mg} / \mathrm{mL}$ ) in water were prepared in separate $25-\mathrm{mL}$ flasks. The $0.50 \mathrm{~mL}$ aliquots of the poly-1-HCl solution $(2.0 \mathrm{mg} / \mathrm{mL})$ were transferred to seven $1-\mathrm{mL}$ flasks equipped with stopcocks. Aliquots of the stock solutions of $(S)$ - and $(R)-2$ were then placed in the flasks so that the percent ee of the mixtures ( $S$-rich) was 5, 10, 20, 40, 60, 80 and 100, and the solutions were finally diluted with water to maintain the poly-1-HCl concentration at $1 \mathrm{mg} / \mathrm{mL}$. After mixing the solutions, the $\mathrm{CD}$ and absorption spectra were measured for each flask (Figure 2B, inset).

Preparation of Liquid Crystal Samples for Polarized Microscopy Studies. The concentrated poly-1-HCl (5 mg) solutions in water (10, 20, 30 and 40 wt. \%; (weight of total polymer $) /[($ weight of total polymer $)+($ weight of water $)])$ were prepared in separate 2-mL test tubes, for the nematic LC poly-1-HCls. After the polymers were completely dissolved to give clear and homogeneous solutions, the solutions were transferred to a $1.0-\mathrm{mm}$ (i.d.) glass capillary with a pipet and the ends were sealed. The cholesteric solutions were prepared in the same way using a $20 \mathrm{wt}$ \% poly-1-HCl solution in water in the presence of appropriate amounts of $(S)$-2 (from 0.0005 to 0.3 equiv.) or 0.1 equiv. nonracemic 2 (from 0.05 to $75 \%$ ee). The samples were left at ambient temperature $\left(20-25^{\circ} \mathrm{C}\right)$ for an hour or days until stable fingerprint textures were obtained. The fingerprint textures showing the retardation lines for the cholesteric LCs were observed using a NIKON E600POL polarized microscope. The fingerprint spacings, which are equal to half of the cholesteric helical pitch, were measured by 
comparing it to a photograph of a standard microscopic ruler.

\section{References}

1. Yashima, E.; Maeda, Y.; Matsushima, T.; Okamoto, Y. Chirality 1997, 9, 593-600.

2. Maeda, K.; Morino, K.; Okamoto, Y.; Sato, T.; Yashima, E. J. Am. Chem. Soc. 2004, 126, 4329-4342.

3. Connors, K. A. Binding Constants; John Wiley: New York, 1987. 


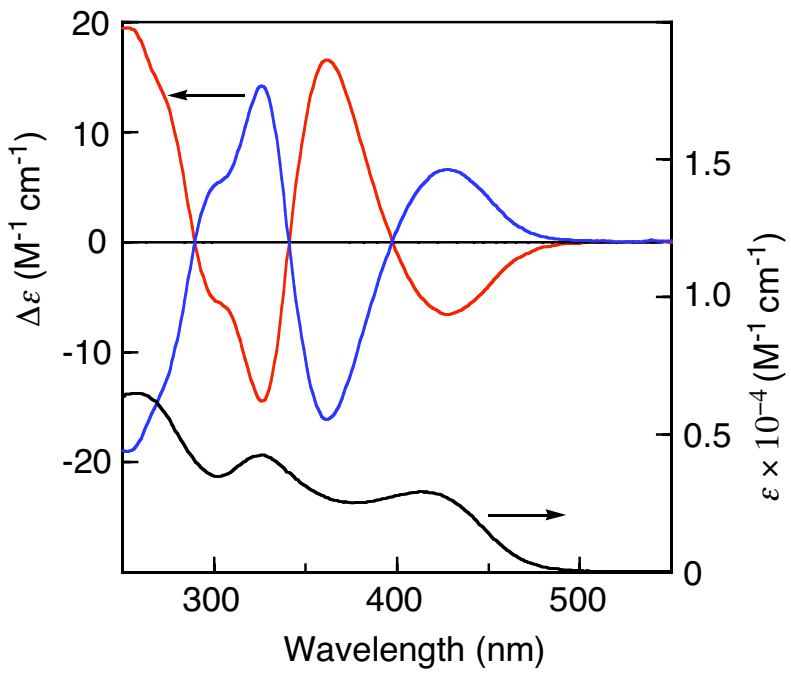

Figure S-1. One-handed helicity induction in poly-1-HCl in water. Shown are the $\mathrm{CD}$ spectra of poly-1-HCl with $(S)$ - (blue line) and $(R)-2$ (red line) and absorption spectrum of poly-1-HCl with $(S)-2$ (black line) in water. Molar ratio of $(S)$ - or $(R)-2$ to the monomeric units of poly-1- $\mathrm{HCl}$ is 0.5 . 